\title{
ON THE ESTIMATION OF FUNCTIONS BELONGING TO LIPSCHITZ CLASS BY BLOCK PULSE FUNCTIONS AND HYBRID LEGENDRE POLYNOMIALS
}

\begin{abstract}
In this paper, block pulse functions and hybrid Legendre polynomials are introduced. The estimators of a function $f$ having first and second derivative belonging to $\operatorname{Lip}_{\alpha}[a, b]$ class, $0<\alpha \leq 1$, and $a, b$ are finite real numbers, by block pulse functions and hybrid Legendre polynomials have been calculated. These calculated estimators are new, sharp and best possible in wavelet analysis. An example has been given to explain the validity of approximation of functions by using the hybrid Legendre polynomials approximation method. A real-world problem of radioactive decay is solved using this hybrid Legendre polynomials approximation method. Moreover, the Hermite differential equation of order zero is solved by using hybrid Legendre polynomials approximation method to explain the importance and the application of the technique of this method.

Key words and phrases: block pulse function, Legendre polynomial, hybrid Legendre polynomial.
\end{abstract}

Institute of Science, Banaras Hindu University, 221005, Varanasi, India

E-mail: shyam_lal@rediffmail.com(Lal S.), vivek.jaimatadi.sharma7@gmail.com (Sharma V.K.)

\section{INTRODUCTION}

In recent years, researchers like Marzban and Razzaghi [8, 10], Hsiao [4] defined and then used hybrid functions (HFs) for the numerical solutions of differential equations and integral equations. Working in the same direction, Marzban et al. [11] derived an operational matrix for a detailed analysis of HFs. In the continuation of their efforts, Merzban [9] studied the optimal control of linear delay systems applying HFs.

Objectives of this research paper are:

(i) to introduce block pulse functions and hybrid Legendre polynomials;

(ii) to estimate the error bounds of the functions of a certain class by hybrid functions;

(iii) to estimate the approximations of a function $f \in \operatorname{Lip}_{\alpha}[a, b]$ by the partial sums of the block function series and hybrid Legendre series.

This research paper is organized as follows. In Section 1, block pulse functions and their some properties, block pulse functions expansion, hybrid Legendre polynomials, hybrid Legendre polynomials expansion, and $\operatorname{Lip}_{\alpha}[a, b]$ class have been explained. In Section 2, the approximation of a function $f \in \operatorname{Lip}_{\alpha}[0,1]$ by block pulse functions expansion, Legendre polynomials expansion and hybrid Legendre polynomials expansion have been estimated and appropriate detailed proofs are provided. In Section 3, hybrid Legendre approximation is explained with the help of an example. Section 4 is introduced to explain the application of this expansion in solving the Hermite differential equation of order zero and in solving some real-world problems. Eventually, some conclusions are mentioned in Section 5. 


\section{DEFINITIONS AND PRELIMINARIES}

\subsection{Block pulse functions and their expansion}

Let $n$ be an arbitrary fixed positive integer. Define functions $\beta_{i}, i=1,2, \ldots, n$, on the interval $[0,1]$ by (see [7])

$$
\beta_{i}(t)= \begin{cases}1, & \frac{i-1}{n} \leq t<\frac{i}{n} \\ 0, & \text { otherwise }\end{cases}
$$

These functions are referred as block pulse functions (or BPFs).

Let $\langle\cdot, \cdot\rangle$ denotes the inner product over the field $F(\mathbb{R}$ or $\mathbb{C})$. Block pulse functions expansion of an $f \in L^{2}[0,1)$ is given by (see [3])

$$
f(t)=\sum_{i=1}^{\infty} f_{\beta_{i}} \beta_{i}(t), \quad f_{\beta_{i}}:=n\left\langle f, \beta_{i}\right\rangle,
$$

where $n$ is an arbitrary fixed positive integer associated with block pulse function $\beta_{i}$. Let $S_{n}$ denotes the $n^{\text {th }}$ partial sum of the series in (1) and it is given by

$$
S_{n}(t)=\sum_{i=1}^{n} f_{\beta_{i}} \beta_{i}(t)
$$

\subsection{Properties of block pulse functions}

An $n$-set of BPFs defined above satisfies the following properties.

1. Disjointness, i.e., $\beta_{i}(t) \beta_{j}(t)=\delta_{i j} \beta_{i}(t)$, where $1 \leq i, j \leq n, \delta_{i j}$ is the Kronecker delta.

2. Orthogonality, i.e.,

$$
\left\langle\beta_{i}, \beta_{j}\right\rangle=\left\{\begin{array}{ll}
0, & i \neq j ; \\
\frac{1}{n}, & i=j,
\end{array} \quad 1 \leq i, j \leq n .\right.
$$

3. Completeness, i.e., for every $f \in L^{2}[0,1)$ Parseval's identity

$$
\int_{0}^{1} f^{2}(t) d t=\left.\sum_{i=1}^{\infty}\left|f_{\beta_{i}}\right|^{2}|| \beta_{i}\right|^{2}
$$

satisfied, where $f_{\beta_{i}}$ is defined in (1).

\subsection{Legendre and hybrid Legendre polynomials}

Legendre differential equation is given by (see [1])

$$
\left(1-x^{2}\right) \frac{d^{2} y}{d x^{2}}-2 x \frac{d y}{d x}+n(n+1) y=0,
$$

where $n$ is a positive integer. Legendre polynomial $L_{n}(x)$ is the solution of above differential equation and it is written in the form (see [2])

$$
L_{n}(x)=\sum_{r=0}^{\left[\frac{n}{2}\right]}(-1)^{r} \frac{(2 n-2 r) !}{2^{n} r !(n-r) !(n-2 r) !} x^{n-2 r}
$$


where

$$
\left[\frac{n}{2}\right]=\left\{\begin{array}{cl}
\frac{n}{2}, & \text { if } n \text { is even; } \\
\frac{n-1}{2}, & \text { if } n \text { is odd. }
\end{array}\right.
$$

Rodrigue' s formula for $L_{n}(x)$ is given by

$$
L_{n}(x)=\frac{1}{2^{n} n !} \frac{d^{n}}{d x^{n}}\left(x^{2}-1\right)^{n}, \quad n=0,1,2, \ldots
$$

Let $n$ and $m$ be the arbitrary fixed positive integers. Hybrid Legendre polynomials, denoted by $h_{i j}, i=1,2, \ldots, n, j=0,1, \ldots, m-1$, on the interval $[0,1)$ are defined by

$$
h_{i j}(t)= \begin{cases}L_{j}(2 n t-2 i+1), & \frac{i-1}{n} \leq t<\frac{i}{n} \\ 0, & \text { otherwise }\end{cases}
$$

where $i$ and $j$ are the orders of BPFs and Legendre polynomials respectively.

\subsection{Hybrid Legendre polynomials expansion}

If $f \in L^{2}[0,1)$, then associated hybrid Legendre polynomial infinite series is (see [6])

$$
f(t)=\sum_{i=1}^{\infty} \sum_{j=0}^{\infty} c_{i j} h_{i j}(t), \quad c_{i j}=\frac{\left\langle f, h_{i j}\right\rangle}{\left\langle h_{i j}, h_{i j}\right\rangle} .
$$

The $(n, m)^{\text {th }}$ partial sums of the series (2) is given by

$$
s_{n, m}(t)=\sum_{i=1}^{n} \sum_{j=0}^{m-1} c_{i j} h_{i j}(t) \text {. }
$$

\section{5 $\operatorname{Lip}_{\alpha}[a, b]$ class}

A function $f$ belongs to $\operatorname{Lip}_{\alpha}[a, b]$ class for $0<\alpha \leq 1$ if

$$
|f(x+t)-f(x)|=O\left(|t|^{\alpha}\right), \quad 0<\alpha \leq 1 .
$$

If $0<\alpha<\beta \leq 1$, then $\operatorname{Lip}_{\beta}[0,1] \subsetneq \operatorname{Lip} \alpha[0,1]$.

Example. Let $\alpha=\frac{1}{3}, \beta=\frac{1}{2}$ and $f(x)=x^{\frac{1}{3}}, g(x)=x^{\frac{1}{2}}, \forall x \in[0,1]$, then $g \in \operatorname{Lip}_{\beta}[0,1] \Rightarrow$ $g \in \operatorname{Lip}_{\alpha}[0,1]$. Here,

$$
|g(x+t)-g(x)|=\left|(x+t)^{\frac{1}{2}}-x^{\frac{1}{2}}\right| \leq|(x+t)-x|^{\frac{1}{2}}=t^{\frac{1}{2}} .
$$

Hence, $|g(x+t)-g(x)|=O\left(t^{\frac{1}{2}}\right)$ and $g \in \operatorname{Lip}_{\frac{1}{2}}[0,1]$. Also,

$$
|g(x+t)-g(x)|=\left|(x+t)^{\frac{1}{2}}-x^{\frac{1}{2}}\right| \leq|(x+t)-x|^{\frac{1}{2}}=t^{\frac{1}{2}} \frac{t^{\frac{1}{3}}}{t^{\frac{1}{3}}}=t^{\frac{1}{3}} t^{\frac{1}{6}} \leq t^{\frac{1}{3}}, \quad \forall t \in[0,1] .
$$

Hence, $|g(x+t)-g(x)|=O\left(t^{\frac{1}{3}}\right)$ and $g \in \operatorname{Lip}_{\frac{1}{3}}[0,1]$. Now,

$$
|f(x+t)-f(x)|=\left|(x+t)^{\frac{1}{3}}-x^{\frac{1}{3}}\right| \leq|(x+t)-x|^{\frac{1}{3}}=t^{\frac{1}{3}}, \quad \forall t \in[0,1] .
$$

Thus, $|f(x+t)-f(x)|=O\left(t^{\frac{1}{3}}\right)$ and $f \in \operatorname{Lip}_{\frac{1}{3}}[0,1]$. But

$$
|f(x+t)-f(x)|=\left|(x+t)^{\frac{1}{3}}-x^{\frac{1}{3}}\right| \leq|(x+t)-x|^{\frac{1}{3}}=t^{\frac{1}{3}} \frac{t^{\frac{1}{2}}}{t^{\frac{1}{2}}}=t^{\frac{1}{2}} t^{-\frac{1}{6}} .
$$

Hence,

$$
\lim _{t \rightarrow 0+}\left|\frac{f(x+t)-f(x)}{t^{\frac{1}{2}}}\right| \rightarrow+\infty .
$$

This shows that $f \notin \operatorname{Lip}_{\frac{1}{2}}[0,1]$. Therefore $\operatorname{Lip}_{\frac{1}{2}}[0,1] \subsetneq \operatorname{Lip}_{\frac{1}{3}}[0,1]$. 


\section{MAin RESUlts}

Theorem 1. Let $f$ be a differentiable function on the interval $[0,1]$ such that its first derivative $f^{\prime} \in \operatorname{Lip}_{\alpha}[0,1]$ and the block pulse functions expansion of $f$ be $f(t)=\sum_{i=1}^{\infty} f_{\beta_{i}} \beta_{i}(t)$, where $f_{\beta_{i}}=\frac{\left\langle f, \beta_{i}\right\rangle}{\left\langle\beta_{i}, \beta_{i}\right\rangle}$, and $\beta_{i}$ is a block pulse function. Then the error of approximation of $f$ by $\left(S_{m} f\right)(t)=\sum_{i=1}^{m} f_{\beta_{i}} \beta_{i}(t)$ is

$$
E^{(B P)}(f)=\min \left\|f-S_{m} f\right\|_{2}=O\left[\frac{1}{m}\left(1+\frac{1}{m^{\alpha}}\right)\right]
$$

where $0<\alpha \leq 1$ and $m$ be an arbitrary fixed positive integer.

Proof. Since

$$
e_{i}=f_{\beta_{i}} \beta_{i}(t)-f(t) \chi_{\left[\frac{i-1}{m}, \frac{i}{m}\right)}, \quad \forall\left[\frac{i-1}{m}, \frac{i}{m}\right), \quad i=1,2, \ldots, m,
$$

where $m$ is an arbitrary fixed positive integer associated with the BPFs and $\chi_{\left[\frac{i-1}{m}, \frac{i}{m}\right)}$ is a characterstic function defined on the interval $\left[\frac{i-1}{m}, \frac{i}{m}\right)$. Then

$$
e_{i}^{2}=f_{\beta_{i}}^{2} \beta_{i}^{2}(t)+f^{2}(t) \chi_{\left[\frac{i-1}{m}, \frac{i}{m}\right)}-2 f_{\beta_{i}} f(t) \chi_{\left[\frac{i-1}{m}, \frac{i}{m}\right)} .
$$

Now, by Taylor theorem (see [5])

$$
\begin{aligned}
\left\|e_{i}\right\|^{2}= & f_{\beta_{i}}^{2} \int_{\frac{i-1}{m}}^{\frac{i}{m}} \beta_{i}^{2}(t) d t+\int_{\frac{i-1}{m}}^{\frac{i}{m}} f^{2}(t) d t-2 f_{\beta_{i}} \int_{\frac{i-1}{m}}^{\frac{i}{m}} \beta_{i}(t) f(t) d t=\frac{f_{\beta_{i}}^{2}}{m}+\int_{\frac{i-1}{m}}^{\frac{i}{m}} f^{2}(t) d t-2 f_{\beta_{i}} \int_{\frac{i-1}{m}}^{\frac{i}{m}} f(t) d t \\
= & \frac{f_{\beta_{i}}^{2}}{m}+\int_{0}^{\frac{1}{m}} f^{2}\left(\frac{i-1}{m}+u\right) d u-2 f_{\beta_{i}} \int_{0}^{\frac{1}{m}} f\left(\frac{i-1}{m}+u\right) d u \\
= & \frac{f_{\beta_{i}}^{2}}{m}+\int_{0}^{\frac{1}{m}}\left\{f^{2}\left(\frac{i-1}{m}\right)+u^{2}\left(f^{\prime}\left(\frac{i-1}{m}+\theta u\right)\right)^{2}\right\} d u \\
& +2 \int_{0}^{\frac{1}{m}} f\left(\frac{i-1}{m}\right) u f^{\prime}\left(\frac{i-1}{m}+\theta u\right) d u-2 f_{\beta_{i}} \int_{0}^{\frac{1}{m}}\left(f\left(\frac{i-1}{m}\right)+u f^{\prime}\left(\frac{i-1}{m}+\theta u\right)\right) d u \\
= & \frac{f_{\beta_{i}}^{2}}{m}+\frac{f^{2}\left(\frac{i-1}{m}\right)}{m}+\int_{0}^{\frac{1}{m}} u^{2}\left(f^{\prime}\left(\frac{i-1}{m}+\theta u\right)\right)^{2} d u \\
& +2 f\left(\frac{i-1}{m}\right) \int_{0}^{\frac{1}{m}} f^{\prime}\left(\frac{i-1}{m}+\theta u\right) u d u-2 f_{\beta_{i}} \frac{f\left(\frac{i-1}{m}\right)}{m}-2 f_{\beta_{i}} \int_{0}^{\frac{1}{m}} u f^{\prime}\left(\frac{i-1}{m}+\theta u\right) d u,
\end{aligned}
$$


where $0<\theta<1$. Also,

$$
\begin{aligned}
f_{\beta_{i}} & =m\left\langle f, \beta_{i}\right\rangle=m \int_{\frac{i-1}{m}}^{\frac{i}{m}} f(t) d t=m \int_{0}^{\frac{1}{m}} f\left(\frac{i-1}{m}+u\right) d u \\
& =m \int_{0}^{\frac{1}{m}}\left(f\left(\frac{i-1}{m}\right)+u f^{\prime}\left(\frac{i-1}{m}+\theta u\right)\right) d u=f\left(\frac{i-1}{m}\right)+m \int_{0}^{\frac{1}{m}} u f^{\prime}\left(\frac{i-1}{m}+\theta u\right) d u .
\end{aligned}
$$

From the above formulas we get

$$
\begin{aligned}
\left\|e_{i}\right\|_{2}^{2}= & \int_{0}^{\frac{1}{m}} u^{2}\left(f^{\prime}\left(\frac{i-1}{m}+\theta u\right)\right)^{2} d u-m\left(\int_{0}^{\frac{1}{m}} u f^{\prime}\left(\frac{i-1}{m}+\theta u\right)\right)^{2} d u \\
= & \int_{0}^{\frac{1}{m}} u^{2}\left\{\left(f^{\prime}\left(\frac{i-1}{m}+\theta u\right)-f^{\prime}\left(\frac{i-1}{m}\right)+f^{\prime}\left(\frac{i-1}{m}\right)\right)\right\}^{2} d u \\
& \quad-m\left(\int_{0}^{\frac{1}{m}} u\left\{\left(f^{\prime}\left(\frac{i-1}{m}+\theta u\right)-f^{\prime}\left(\frac{i-1}{m}\right)+f^{\prime}\left(\frac{i-1}{m}\right)\right)\right\}\right)^{2} d u \\
\leq & \int_{0}^{\frac{1}{m}} u^{2}\left(A_{1} u^{\alpha}+f^{\prime}\left(\frac{i-1}{m}\right)\right)^{2} d u+m\left(\int_{0}^{\frac{1}{m}} u\left\{A_{1} u^{\alpha}+f^{\prime}\left(\frac{i-1}{m}\right)\right\} d u\right)^{2} \\
= & \frac{A_{1}^{2}}{m^{2 \alpha+3}}\left(\frac{1}{2 \alpha+3}+\frac{1}{(\alpha+2)^{2}}\right)+\frac{\left(f^{\prime}\left(\frac{i-1}{m}\right)\right)^{2}}{12 m^{3}}+\frac{2 A_{1} f^{\prime}\left(\frac{i-1}{m}\right)}{m^{\alpha+3}}\left(\frac{1}{\alpha+3}+\frac{1}{2 \alpha+4}\right),
\end{aligned}
$$

where $A_{1}$ is a positive constant.

Hence,

$$
\begin{aligned}
\|e\|_{2}^{2}=\sum_{i=1}^{m}\left\|e_{i}\right\|_{2}^{2} & =\frac{A_{1}^{2}}{m^{2 \alpha+2}}\left(\frac{1}{2 \alpha+3}+\frac{1}{(\alpha+2)^{2}}\right)+\frac{\left(f^{\prime}\left(\frac{i-1}{m}\right)\right)^{2}}{12 m^{2}}+\frac{2 A_{1} f^{\prime}\left(\frac{i-1}{m}\right)}{m^{\alpha+2}}\left(\frac{1}{\alpha+3}+\frac{1}{2 \alpha+4}\right) \\
& \leq \frac{2 A_{1}^{2}}{m^{2 \alpha+2}}+\frac{\left(f^{\prime}\left(\frac{i-1}{m}\right)\right)^{2}}{12 m^{2}}+\frac{4 A_{1} f^{\prime}\left(\frac{i-1}{m}\right)}{m^{\alpha+2}} \\
& =2\left(\frac{A_{1}^{2}}{m^{2 \alpha+2}}+\frac{\left(f^{\prime}\left(\frac{i-1}{m}\right)\right)^{2}}{24 m^{2}}+\frac{2 A_{1} f^{\prime}\left(\frac{i-1}{m}\right)}{m^{\alpha+2}}\right) \leq 2\left(\frac{A_{1}}{m^{\alpha+1}}+\frac{f^{\prime}\left(\frac{i-1}{m}\right)}{m}\right)^{2} .
\end{aligned}
$$

Therefore,

$$
\|e\|_{2}=O\left[\frac{1}{m}\left(1+\frac{1}{m^{\alpha}}\right)\right] .
$$

So, the proof of Theorem 1 is completely established.

Theorem 2. Let $f$ be a differentiable function defined on the interval $[-1,1]$ such that its second derivative $f^{\prime \prime} \in \operatorname{Lip} \alpha[-1,1]$ and Legendre expansion of function $f$ be

$$
f(t)=\sum_{j=0}^{\infty} c_{j} P_{j}(t)
$$

where $P_{j}$ is a Legendre polynomial and $c_{j}=\frac{\left\langle f, P_{j}\right\rangle}{\left\langle P_{j}, P_{j}\right\rangle}$. Then the error of the approximation of $f$ $\begin{aligned} \text { by }\left(U_{m} f\right)(t)= & \sum_{j=0}^{m-1} c_{j} P_{j}(t), m=1,2, \ldots, \text { is } \\ & E^{(L P)}(f)=\min \left\|f-U_{m} f\right\|_{2} \leq M\left(1+\frac{1}{\sqrt{2 \alpha+1}}\right) \frac{1}{(2 m-3)^{\frac{3}{2}}},\end{aligned}$ 
where $m \geq 2$ is an integer, $M$ is a positive constant and $0<\alpha \leq 1$.

Proof. Legendre expansion of a function $f(t)$ is given by (3). Let

$$
U_{m}(t)=\sum_{j=0}^{m-1} c_{j} P_{j}(t)
$$

denotes the $m^{\text {th }}$ partial sum of (3). Then for arbitrary $m \geq 2$, we have

$$
\begin{aligned}
\left\|f-U_{m}\right\|_{2}^{2}=\int_{-1}^{1}\left(f-U_{m}\right)^{2} d t & =\int_{-1}^{1}\left(\sum_{j=0}^{m-1} c_{j} P_{j}(t)\right)^{2} d t \\
& =\sum_{j=m}^{\infty} c_{j}^{2} \int_{-1}^{1} P_{j}^{2} d t=\sum_{j=m}^{\infty} c_{j}^{2}\left(\frac{2}{2 j+1}\right),
\end{aligned}
$$

and for arbitrary $j \geq m$, we obtain

$$
\begin{aligned}
& c_{j}= \frac{\int_{-1}^{1} f(t) P_{j}(t) d t}{\int_{-1}^{1} P_{j}^{2} d t}=\frac{2 j+1}{2} \int_{-1}^{1} f(t) P_{j}(t) d t=\frac{2 j+1}{2} \int_{-1}^{1} f(t) \frac{P_{j+1}^{\prime}-P_{j-1}^{\prime}}{2 j+1} d t \\
&= \frac{1}{2} \int_{-1}^{1} f(t)\left(P_{j+1}^{\prime}-P_{j-1}^{\prime}\right) d t=\frac{1}{2} \int_{-1}^{1} f^{\prime}(t)\left(P_{j-1}-P_{j+1}\right) d t \\
&= \frac{1}{2} \int_{-1}^{1} f^{\prime}(t) P_{j-1} d t-\int_{-1}^{1} f^{\prime}(t) P_{j+1} d t=\frac{1}{2(2 j-1)} \int_{-1}^{1} f^{\prime}(t)\left(P_{j}^{\prime}-P_{j-2}^{\prime}\right) d t \\
& \frac{1}{2(2 j+3)} \int_{-1}^{1} f^{\prime}(t)\left(P_{j+2}^{\prime}-P_{j}^{\prime}\right) d t \\
& \frac{1}{2(2 j-1)} \int_{-1}^{\prime \prime} f^{\prime \prime}(t)\left(P_{j-2}-P_{j}\right) d t+\frac{1}{2(2 j+3)} \int_{-1}^{1} f^{\prime \prime}(t)\left(P_{j+2}-P_{j}\right) d t=\frac{1}{2} \int_{-1}^{1} f^{\prime \prime}(t) B_{j}(t) d t,
\end{aligned}
$$

where $B_{j}(t)=\frac{P_{j-2}-P_{j}}{2 j-1}+\frac{P_{j+2}-P_{j}}{2 j+3}$. Hence,

$$
\begin{aligned}
\left|c_{j}\right| & \leq \frac{1}{2} \int_{-1}^{1}\left|f^{\prime \prime}(t)-f^{\prime \prime}(0)\right|\left|B_{j}(t)\right| d t+\frac{1}{2} \int_{-1}^{1}\left|f^{\prime \prime}(0)\right|\left|B_{j}(t)\right| d t \\
& \leq \frac{M_{1}}{2} \int_{-1}^{1}|t|^{\alpha}\left|B_{j}(t)\right| d t+\frac{\left|f^{\prime \prime}(0)\right|}{2} \int_{-1}^{1}\left|B_{j}(t)\right| d t
\end{aligned}
$$


where $M_{1}$ is a positive constant. Next, applying the Cauchy-Schwarz inequality, we get

$$
\begin{aligned}
\left(\int_{-1}^{1}|t|^{\alpha}\left|B_{j}\right| d t\right)^{2} & \leq \int_{-1}^{1}|t|^{2 \alpha} d t \int_{-1}^{1}\left|B_{j}\right|^{2} d t=2 \int_{0}^{1}|t|^{2 \alpha} d t \int_{-1}^{1}\left|B_{j}\right|^{2} d t \\
& =\frac{2}{2 \alpha+1} \int_{-1}^{1}\left(\frac{P_{j+2}^{2}+P_{j}^{2}}{(2 j+3)^{2}}+\frac{P_{j-2}^{2}+P_{j}^{2}}{(2 j-1)^{2}}\right) d t \\
& =\frac{2}{(2 \alpha+1)(2 j+3)^{2}}\left(\frac{2}{2 j+5}+\frac{2}{2 j+1}\right)+\frac{2}{(2 \alpha+1)(2 j-1)^{2}}\left(\frac{2}{2 j-3}+\frac{2}{2 j+1}\right) \\
& \leq \frac{16}{(2 \alpha+1)(2 j-3)^{3}} .
\end{aligned}
$$

Hence,

$$
\int_{-1}^{1}|t|^{\alpha}\left|B_{j}\right| d t \leq \frac{4}{\sqrt{2 \alpha+1}(2 j-3)^{\frac{3}{2}}}
$$

Also,

$$
\left(\int_{-1}^{1}\left|B_{j}\right| d t\right)^{2} \leq\left(\int_{-1}^{1} 1^{2} d t\right)^{2}\left(\int_{-1}^{1}\left|B_{j}\right|^{2} d t\right) \leq \frac{16}{(2 j-3)^{3}}
$$

Therefore,

$$
\int_{-1}^{1}\left|B_{j}\right| d t \leq \frac{4}{(2 j-3)^{\frac{3}{2}}}
$$

By (4)-(6) we have

$$
\left|c_{j}\right| \leq \frac{1}{2} \frac{4 M_{1}}{\sqrt{2 \alpha+1}} \frac{1}{(2 j-3)^{\frac{3}{2}}}+\frac{\left|f^{\prime \prime}(0)\right|}{2} \frac{4}{(2 j-3)^{\frac{3}{2}}} \leq M\left(\frac{1}{\sqrt{2 \alpha+1}}+1\right) \frac{1}{(2 j-3)^{\frac{3}{2}}},
$$

where $M=\max \left\{2 M_{1}, 2\left|f^{\prime \prime}(0)\right|\right\}$ and

$$
\begin{aligned}
\left\|f-U_{m}\right\|_{2}^{2} & \leq \sum_{j=m}^{\infty} M^{2}\left(1+\frac{1}{\sqrt{2 \alpha+1}}\right)^{2} \frac{1}{(2 j-3)^{3}} \frac{2}{(2 j+1)} \\
& \leq 2 M^{2}\left(1+\frac{1}{\sqrt{2 \alpha+1}}\right)^{2} \sum_{j=m}^{\infty} \frac{1}{(2 j-3)^{4}} \\
& \leq 2 M^{2}\left(1+\frac{1}{\sqrt{2 \alpha+1}}\right)^{2} \frac{1}{(2 m-3)^{3}}, \quad m \geq 2 .
\end{aligned}
$$

Hence,

$$
\left\|f-U_{m}\right\|_{2} \leq \sqrt{2} M\left(1+\frac{1}{\sqrt{2 \alpha+1}}\right) \frac{1}{(2 m-3)^{\frac{3}{2}}} .
$$

Thus Theorem 2 is completely proved. 
Theorem 3. Let $f$ be a differentiable function on $[0,1]$ such that $f^{\prime \prime} \in \operatorname{Lip} p_{\alpha}[0,1]$ and hybrid Legendre polynomials expansion of $f$ be

$$
f(t)=\sum_{i=1}^{\infty} \sum_{j=0}^{\infty} c_{i j} h_{i j}(t)
$$

where $c_{i j}=\frac{\left\langle f, h_{i j}\right\rangle}{\left\langle h_{i j}, h_{i j}\right\rangle}$ and $h_{i j}$ is the hybrid Legendre polynomials, and

$$
\left(S_{n, m} f\right)(t)=\sum_{i=1}^{n} \sum_{j=0}^{m-1} c_{i j} h_{i j}(t)
$$

be the $(n, m)^{\text {th }}$ partial sum of the series (7). Then the error of approximation $f$ by $S_{n, m} f$ is

$$
E_{n, m}^{(H F s)} f=\min \left\|f-S_{n, m} f\right\|_{2}=O\left[\left(\frac{1}{n^{\alpha+2}}+\frac{1}{2 n^{2}}\right) \frac{1}{(2 m-3)^{\frac{3}{2}}}\right],
$$

where $m \geq 2, n$ is a positive integer and $0<\alpha \leq 1$.

Proof. We see that hybrid Legendre polynomials expansion of $f$ is given by (7). Now, suppose $n$ and $m$ are the arbitrary fixed positive integers. Then for $i=1,2, \ldots, n$ and $j=0,1, \ldots, m-1$, we have

$$
\begin{aligned}
c_{i j}= & \frac{\left\langle f, h_{i j}\right\rangle}{\left\langle h_{i j}, h_{i j}\right\rangle}=\frac{\int_{\frac{i-1}{n}}^{\frac{i}{n}} f(t) h_{i j}(t) d t}{\int_{\frac{i-1}{n}}^{\frac{i}{n}} h_{i j}^{2}(t) d t}=\frac{\int_{\frac{i-1}{n}}^{\frac{i}{n}} f(t) P_{j}(2 n t-2 i+1) d t}{\int_{\frac{i-1}{n}}^{\frac{i}{n}} P_{j}^{2}(2 n t-2 i+1) d t} \\
= & \frac{\int_{-1}^{1} f\left(\frac{u+2 i-1}{2 n}\right) P_{j}(u) \frac{d u}{2 n}}{\int_{-1}^{1} P_{j}^{2}(u) \frac{d u}{2 n}}=\frac{2 j+1}{2} \int_{-1}^{1} f\left(\frac{u+2 i-1}{2 n}\right) P_{j}(u) d u \\
= & \frac{2 j+1}{2} \int_{-1}^{1} f\left(\frac{t+2 i-1}{2 n}\right) P_{j}(t) d t=\frac{2 j+1}{2} \int_{-1}^{1} f\left(\frac{t+2 i-1}{2 n}\right)\left(\frac{P_{j+1}^{\prime}-P_{j-1}^{\prime}}{2 j+1}\right) d t \\
= & \frac{1}{2} \int_{-1}^{1} f\left(\frac{t+2 i-1}{2 n}\right)\left(P_{j+1}^{\prime}-P_{j-1}^{\prime}\right) d t=\frac{-1}{4 n} \int_{-1}^{\prime} f^{\prime}\left(\frac{t+2 i-1}{2 n}\right)\left(P_{j+1}-P_{j-1}\right) d t \\
= & \frac{-1}{4 n} \int_{-1}^{1} f^{\prime}\left(\frac{t+2 i-1}{2 n}\right) P_{j+1} d t+\frac{1}{4 n} \int_{-1}^{1} f^{\prime}\left(\frac{t+2 i-1}{2 n}\right) P_{j-1} d t=I_{1}+I_{2} .
\end{aligned}
$$


Now,

$$
\begin{aligned}
I_{1} & =\frac{-1}{4 n} \int_{-1}^{1} f^{\prime}\left(\frac{t+2 i-1}{2 n}\right) P_{j+1} d t=\frac{-1}{4 n} \int_{-1}^{1} f^{\prime}\left(\frac{t+2 i-1}{2 n}\right)\left(\frac{P_{j+2}^{\prime}-P_{j}^{\prime}}{2 j+3}\right) d t \\
& =\frac{1}{8 n^{2}(2 j+3)} \int_{-1}^{1} f^{\prime \prime}\left(\frac{t+2 i-1}{2 n}\right)\left(P_{j+2}-P_{j}\right) d t \\
& =\frac{1}{8 n^{2}} \int_{-1}^{1} f^{\prime \prime}\left(\frac{t+2 i-1}{2 n}\right)\left(\frac{P_{j+2}-P_{j}}{2 j+3}\right) d t .
\end{aligned}
$$

Next,

$$
I_{2}=\frac{1}{4 n} \int_{-1}^{1} f^{\prime}\left(\frac{t+2 i-1}{2 n}\right) P_{j-1} d t=\frac{1}{8 n^{2}} \int_{-1}^{1} f^{\prime \prime}\left(\frac{t+2 i-1}{2 n}\right)\left(\frac{P_{j-2}-P_{j}}{2 j-1}\right) d t .
$$

By (8)-(10) we have

$$
\begin{aligned}
c_{i j} & =\frac{1}{8 n^{2}} \int_{-1}^{1} f^{\prime \prime}\left(\frac{t+2 i-1}{2 n}\right)\left(\frac{P_{j+2}-P_{j}}{2 j+3}\right) d t+\frac{1}{8 n^{2}} \int_{-1}^{1} f^{\prime \prime}\left(\frac{t+2 i-1}{2 n}\right)\left(\frac{P_{j-2}-P_{j}}{2 j-1}\right) d t \\
& =\frac{1}{8 n^{2}} \int_{-1}^{1} f^{\prime \prime}\left(\frac{t+2 i-1}{2 n}\right)\left(\frac{P_{j+2}-P_{j}}{2 j+3}+\frac{P_{j-2}-P_{j}}{2 j-1}\right) d t=\frac{1}{8 n^{2}} \int_{-1}^{1} f^{\prime \prime}\left(\frac{t+2 i-1}{2 n}\right) B_{j} d t,
\end{aligned}
$$

where $B_{j}=\frac{P_{j+2}-P_{j}}{2 j+3}+\frac{P_{j-2}-P_{j}}{2 j-1}$. Hence,

$$
\begin{aligned}
\left|c_{i j}\right| & \leq \frac{1}{8 n^{2}} \int_{-1}^{1}\left|f^{\prime \prime}\left(\frac{t+2 i-1}{2 n}\right)\right|\left|B_{j}\right| d t \\
& \leq \frac{1}{8 n^{2}} \int_{-1}^{1}\left|f^{\prime \prime}\left(\frac{t+2 i-1}{2 n}\right)-f^{\prime \prime}\left(\frac{2 i-1}{2 n}\right)\right|\left|B_{j}\right| d t+\frac{1}{8 n^{2}} \int_{-1}^{1}\left|f^{\prime \prime}\left(\frac{2 i-1}{2 n}\right)\right|\left|B_{j}\right| d t \\
& \leq \frac{M_{1}}{2^{\alpha+3} n^{\alpha+2}} \int_{-1}^{1}|t|^{\alpha}\left|B_{j}\right| d t+\frac{1}{8 n^{2}}\left|f^{\prime \prime}\left(\frac{2 i-1}{2 n}\right)\right| \int_{-1}^{1}\left|B_{j}\right| d t
\end{aligned}
$$

where $M_{1}$ is a positive constant.

Now, by (5), (6) and (11) we get

$$
\begin{aligned}
\left|c_{i j}\right| & \leq \frac{M_{1}}{2^{\alpha+3} n^{\alpha+2}} \frac{4}{\sqrt{2 \alpha+1}(2 j-3)^{\frac{3}{2}}}+\frac{1}{8 n^{2}}\left|f^{\prime \prime}\left(\frac{2 i-1}{2 n}\right)\right| \frac{4}{(2 j-3)^{\frac{3}{2}}} \\
& \leq \frac{M_{1}}{2^{\alpha+3} n^{\alpha+2}} \frac{4}{\sqrt{2 \alpha+1}(2 j-3)^{\frac{3}{2}}}+\frac{1}{8 n^{2}} M_{2} \frac{4}{(2 j-3)^{\frac{3}{2}}} \\
& \leq B\left(\frac{1}{\sqrt{2 \alpha+1} 2^{\alpha+1} n^{\alpha+2}}+\frac{1}{2 n^{2}}\right) \frac{1}{(2 j-3)^{\frac{3}{2}}} \leq B\left(\frac{1}{n^{\alpha+2}}+\frac{1}{2 n^{2}}\right) \frac{1}{(2 j-3)^{\frac{3}{2}}}
\end{aligned}
$$


where $B=\max \left\{M_{1}, M_{2}\right\}$. Since $f^{\prime \prime} \in \operatorname{Lip}_{\alpha}[0,1], 0<\alpha \leq 1$, it is continuous on $[0,1]$. Therefore it is bounded on $[0,1]$. Thus, there exists a constant $M_{2}$ independent of $t$ such that $\left|f^{\prime \prime}(t)\right| \leq M_{2}$ $\forall t \in[0,1]$. Also $n \in \mathbb{N}$ and $i=1,2, \ldots, n$, so $0 \leq \frac{i-1}{n}<1$, i.e. $\frac{i-1}{n} \in[0,1]$ for each $n$ and $i=1,2, \ldots, n$. Hence $\left|f^{\prime \prime}\left(\frac{i-1}{n}\right)\right| \leq M_{2}$.

Let $S_{n, m} f$ denotes the $(n, m)^{t h}$ partial sum of the series (7) as given in theorem 3 . Now,

$$
f-S_{n, m} f=\left(\sum_{i=1}^{n}+\sum_{i=n+1}^{\infty}\right)\left(\sum_{j=0}^{m-1}+\sum_{j=m}^{\infty}\right) c_{i j} h_{i j}-\sum_{i=1}^{n} \sum_{j=0}^{m-1} c_{i j} h_{i j}=\sum_{i=1}^{n} \sum_{j=m}^{\infty} c_{i j} h_{i j} .
$$

Then

$$
\begin{aligned}
\left\|f-S_{n, m} f\right\|_{2}^{2} & =\int_{0}^{1}\left(\sum_{i=1}^{n} \sum_{j=m}^{\infty} c_{i j} h_{i j}\right)^{2} d t=\sum_{i=1}^{n} \sum_{j=m}^{\infty} c_{i j}^{2} \int_{0}^{1} h_{i j}^{2} d t \\
& =\sum_{i=1}^{n} \sum_{j=m}^{\infty} c_{i j}^{2} \int_{\frac{i-1}{m}}^{\frac{i}{m}} P_{j}^{2}(2 n t-2 i+1) d t=\sum_{i=1}^{n} \sum_{j=m}^{\infty} c_{i j}^{2} \frac{1}{n(2 j+1)} .
\end{aligned}
$$

By (12) and (13) we have

$$
\begin{aligned}
\left\|f-S_{n, m} f\right\|_{2}^{2} & \leq \sum_{i=1}^{n} \sum_{j=m}^{\infty} B^{2}\left(\frac{1}{n^{\alpha+2}}+\frac{1}{2 n^{2}}\right)^{2} \frac{1}{(2 j-3)^{3}} \frac{1}{n(2 j+1)} \\
& =B^{2}\left(\frac{1}{n^{\alpha+2}}+\frac{1}{2 n^{2}}\right)^{2} \sum_{j=m}^{\infty} \frac{1}{(2 j-3)^{3}} \frac{1}{(2 j+1)} \\
& \leq B^{2}\left(\frac{1}{n^{\alpha+2}}+\frac{1}{2 n^{2}}\right)^{2} \sum_{j=m}^{\infty} \frac{1}{(2 j-3)^{4}} \leq B^{2}\left(\frac{1}{n^{\alpha+2}}+\frac{1}{2 n^{2}}\right)^{2} \frac{1}{(2 m-3)^{3}} .
\end{aligned}
$$

Hence,

$$
\left\|f-S_{n, m} f\right\|_{2}^{2} \leq B\left(\frac{1}{n^{\alpha+2}}+\frac{1}{2 n^{2}}\right) \frac{1}{(2 m-3)^{\frac{3}{2}}} .
$$

Therefore,

$E_{n, m}^{(H F s)}(f)=\min \left\|f-S_{n, m} f\right\|_{2}=O\left[\left(\frac{1}{n^{\alpha+2}}+\frac{1}{2 n^{2}}\right) \frac{1}{(2 m-3)^{\frac{3}{2}}}\right], \quad m \geq 2$ and $0<\alpha \leq 1$.

\section{NUMERICAL EXAMPLE OF HYBRID LEGENDRE POLYNOMIALS APPROXIMATION}

In this section hybrid Legendre polynomials approximation of the function

$$
f(t)= \begin{cases}t^{3}+t^{2}+2 t+1, & \forall t \in[0,1] \\ 0, & \text { otherwise }\end{cases}
$$


for $n=1,2$ and $m=1,2,3$ has been explained by graphs of concerned function. $S_{n, m}$ for $n=1,2$ and $m=1,2,3$ are calculated and are given as

$$
\begin{aligned}
& S_{1,1}(t)=\left\{\begin{array}{ll}
\frac{31}{12}, & 0 \leq t<1, \\
0, & \text { otherwise, }
\end{array} \quad S_{1,2}(t)= \begin{cases}\frac{31}{12}+\frac{39}{12}(2 t-1), & 0 \leq t<1, \\
0, & \text { otherwise, }\end{cases} \right. \\
& S_{1,3}(t)= \begin{cases}\frac{31}{12}+\frac{39}{12}(2 t-1)+\frac{5}{24}\left[3(2 t-1)^{2}-1\right], & 0 \leq t<1, \\
0, & \text { otherwise, }\end{cases} \\
& S_{2,1}(t)=\left\{\begin{array}{ll}
\frac{155}{96}, & 0 \leq t<\frac{1}{2}, \\
\frac{341}{96}, & \frac{1}{2} \leq t<1, \\
0, & \text { otherwise, }
\end{array} \quad S_{2,2}(t)= \begin{cases}\frac{155}{96}+\frac{109}{160}(4 t-1), & 0 \leq t<\frac{1}{2}, \\
\frac{341}{96}+\frac{209}{160}(4 t-3), & \frac{1}{2} \leq t<1, \\
0, & \text { otherwise, }\end{cases} \right. \\
& S_{2,3}(t)= \begin{cases}\frac{155}{96}+\frac{109}{160}(4 t-1)+\frac{7}{192}\left[3(4 t-1)^{2}-1\right], & 0 \leq t<\frac{1}{2} \\
\frac{341}{96}+\frac{209}{160}(4 t-3)+\frac{13}{192}\left[3(4 t-3)^{2}-1\right], & \frac{1}{2} \leq t<1 \\
0, & \text { otherwise }\end{cases}
\end{aligned}
$$

The graphs of $S_{n, m}$ and $f(t)$ has been plotted for $n=1,2$ and $m=1,2,3$ in Figures 1-6 respectively. Hybrid Legendre polynomial approximation error for different values of $n$ and $m$ is shown in Table 1.

\begin{tabular}{|c|c|c|}
\hline $\mathrm{n}$ & $\mathrm{m}$ & $\left\|f-S_{n, m}\right\|_{2}$ \\
\hline \multirow{3}{*}{$n=1$} & $\mathrm{~m}=1$ & 1.14131 \\
\cline { 2 - 3 } & $\mathrm{m}=2$ & 0.187295 \\
\cline { 2 - 3 } & $\mathrm{m}=3$ & 0.0188982 \\
\hline \multirow{3}{*}{$n=2$} & $\mathrm{~m}=1$ & 0.603409 \\
\cline { 2 - 3 } & $\mathrm{m}=2$ & 0.0486932 \\
\cline { 2 - 3 } & $\mathrm{m}=3$ & 0.00236228 \\
\hline
\end{tabular}

Table 1. Hybrid Legendre polynomial approximation errors for different values of $n$ and $m$

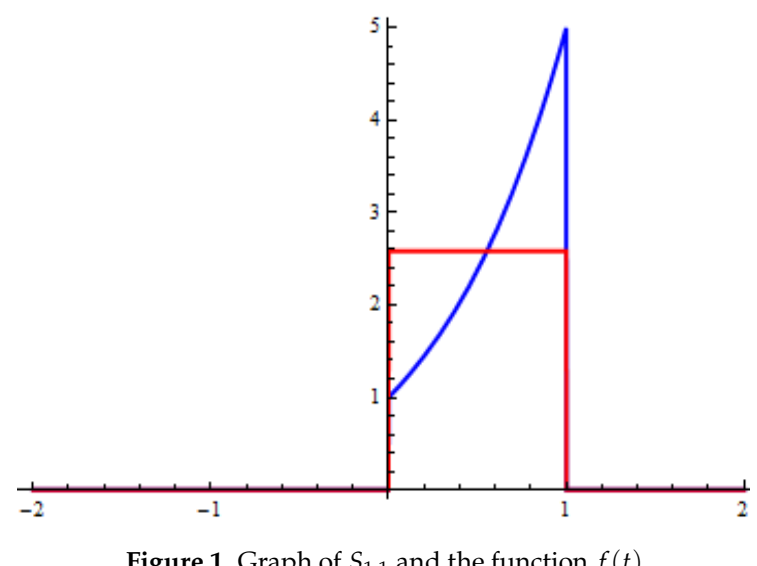

Figure 1. Graph of $S_{1,1}$ and the function $f(t)$

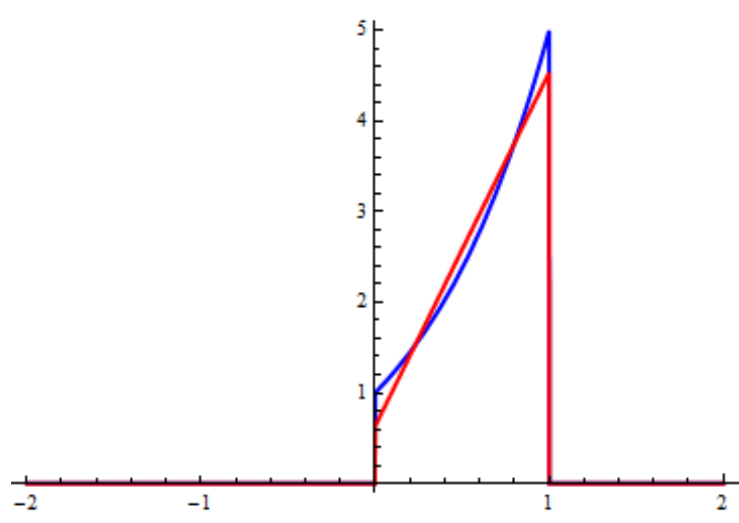

Figure 2. Graph of $S_{1,2}$ and the function $f(t)$ 

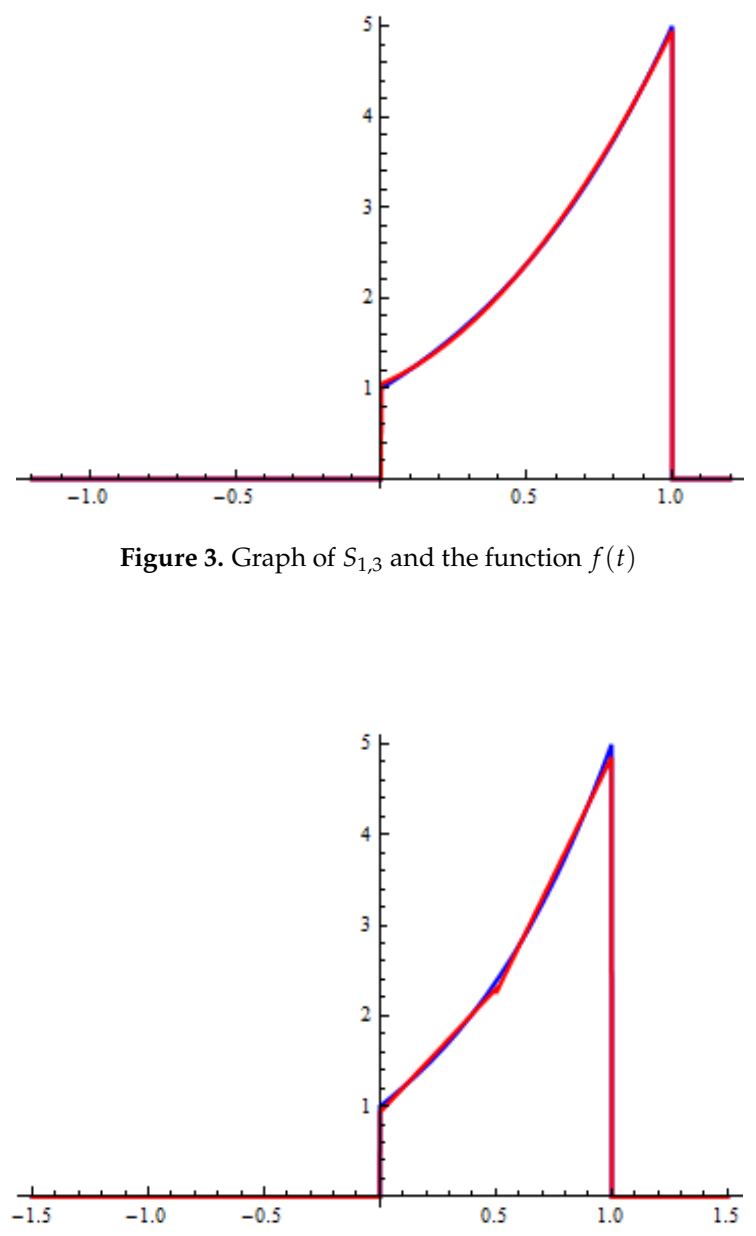

Figure 5. Graph of $S_{2,2}$ and the function $f(t)$
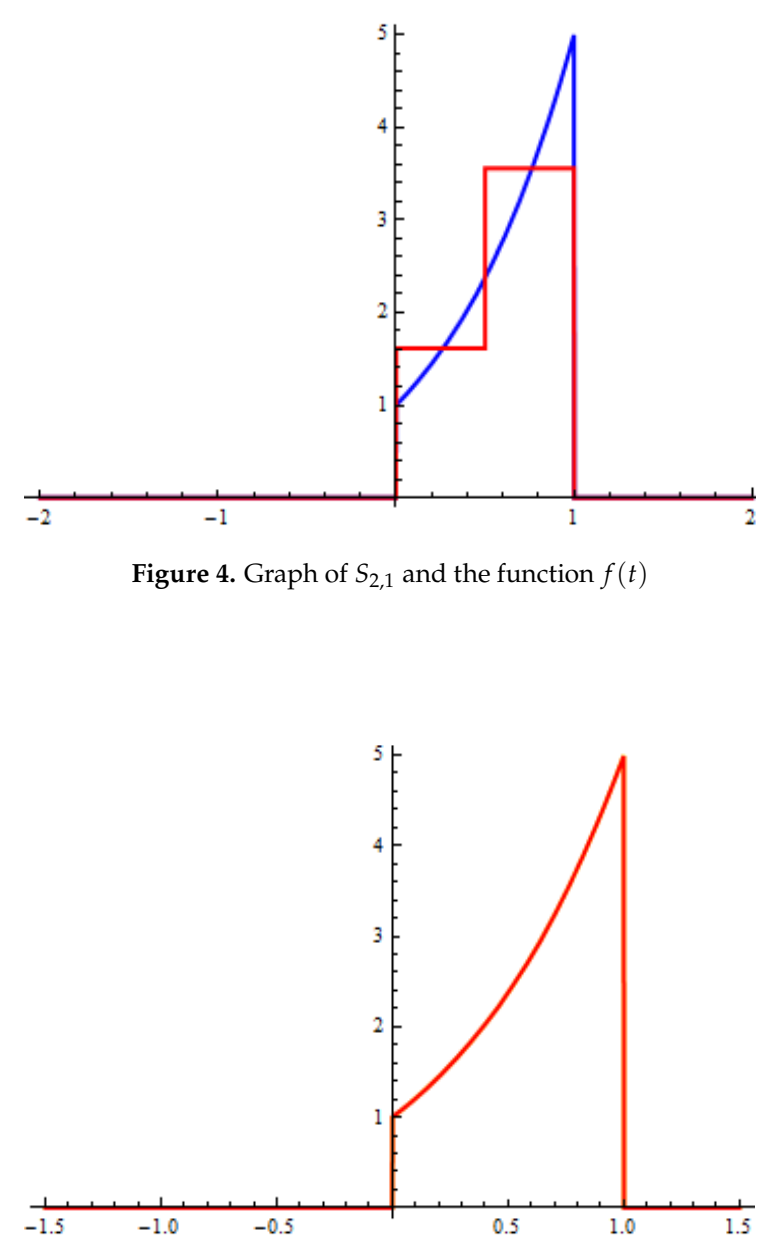

Figure 6. Graph of $S_{2,3}$ and the function $f(t)$

\section{APPLICATION OF HYBRID LEGENDRE POLYNOMIALS EXPANSION}

\subsection{Application of hybrid Legendre polynomials expansion in real-world problems}

We have used the hybrid Legendre polynomial approximation method to solve the differential equations related to the following real-world problem.

\subsubsection{Radioactive decay}

Radioactivity [12] is one of the effects of disruption in the nucleus of a radioactive substance. It is important to remember that radioactivity has also been used in the diagnosis of cancers through lighting in the nucleus form of the atoms to the recipient.

If $m(t)$ be the mass of a radioactive substance at time $t$, then (see [12])

$$
\frac{d m}{d t}=-k m(t), \quad m(0)=m_{0}
$$

where $k$ is a decay constant and $m_{0}$ is the initial mass. Let us consider $k=2$ and $m_{0}=2$, the above equation reduces to

$$
\frac{d m}{d t}=-2 m(t), \quad m(0)=2
$$


Equation (15) is now solved using hybrid Legendre polynomials operational matrix of integration as in [6] for $n=5$ and $m=3$ as below.

Let

$$
h(t)=\left[h_{10}, h_{11}, h_{12}, h_{20}, h_{21}, h_{22}, h_{30}, h_{31}, h_{32}, h_{40}, h_{41}, h_{42}, h_{50}, h_{51}, h_{52}\right]^{T} .
$$

Here $h(t)$ be $15 \times 1$ column vector and $h_{i j}$ for $i=1,2,3,4,5$ and $j=0,1,2$ are calculated as given in subsection 1.3. The integration of above vector $h(t)$ is given as

$$
\int_{0}^{t} h(x) d x=P h(t)
$$

Here $P$ is $15 \times 15$ hybrid Legendre polynomials operational matrix of integration and it is given as

$$
P=\left[\begin{array}{ccccccccccccccc}
\frac{1}{10} & \frac{1}{10} & 0 & \frac{1}{5} & 0 & 0 & \frac{1}{5} & 0 & 0 & \frac{1}{5} & 0 & 0 & \frac{1}{5} & 0 & 0 \\
-\frac{1}{30} & 0 & \frac{1}{30} & 0 & 0 & 0 & 0 & 0 & 0 & 0 & 0 & 0 & 0 & 0 & 0 \\
0 & -\frac{1}{50} & 0 & 0 & 0 & 0 & 0 & 0 & 0 & 0 & 0 & 0 & 0 & 0 & 0 \\
0 & 0 & 0 & \frac{1}{10} & \frac{1}{10} & 0 & \frac{1}{5} & 0 & 0 & \frac{1}{5} & 0 & 0 & \frac{1}{5} & 0 & 0 \\
0 & 0 & 0 & -\frac{1}{30} & 0 & \frac{1}{30} & 0 & 0 & 0 & 0 & 0 & 0 & 0 & 0 & 0 \\
0 & 0 & 0 & 0 & -\frac{1}{50} & 0 & 0 & 0 & 0 & 0 & 0 & 0 & 0 & 0 & 0 \\
0 & 0 & 0 & 0 & 0 & 0 & \frac{1}{10} & \frac{1}{10} & 0 & \frac{1}{5} & 0 & 0 & \frac{1}{5} & 0 & 0 \\
0 & 0 & 0 & 0 & 0 & 0 & -\frac{1}{30} & 0 & \frac{1}{30} & 0 & 0 & 0 & 0 & 0 & 0 \\
0 & 0 & 0 & 0 & 0 & 0 & 0 & -\frac{1}{50} & 0 & 0 & 0 & 0 & 0 & 0 & 0 \\
0 & 0 & 0 & 0 & 0 & 0 & 0 & 0 & 0 & \frac{1}{10} & \frac{1}{10} & 0 & \frac{1}{5} & 0 & 0 \\
0 & 0 & 0 & 0 & 0 & 0 & 0 & 0 & 0 & -\frac{1}{30} & 0 & \frac{1}{30} & 0 & 0 & 0 \\
0 & 0 & 0 & 0 & 0 & 0 & 0 & 0 & 0 & 0 & -\frac{1}{50} & 0 & 0 & 0 & 0 \\
0 & 0 & 0 & 0 & 0 & 0 & 0 & 0 & 0 & 0 & 0 & 0 & \frac{1}{10} & \frac{1}{10} & 0 \\
0 & 0 & 0 & 0 & 0 & 0 & 0 & 0 & 0 & 0 & 0 & 0 & -\frac{1}{30} & 0 & \frac{1}{30} \\
0 & 0 & 0 & 0 & 0 & 0 & 0 & 0 & 0 & 0 & 0 & 0 & 0 & -\frac{1}{50} & 0
\end{array}\right] .
$$

Let $m(t)=N^{T} h(t)$, where

$$
N(t)=\left[n_{10}, n_{11}, n_{12}, n_{20}, n_{21}, n_{22}, n_{30}, n_{31}, n_{32}, n_{40}, n_{41}, n_{42}, n_{50}, n_{51}, n_{52}\right]^{T}
$$

is an unknown vector. Integrating equation (15) and using initial conditions, we observe

$$
\left(I+2 P^{T}\right) N=2 d .
$$

Here $I$ be a identity matrix of order 15 and $d=[1,0,0,1,0,0,1,0,0,1,0,0,1,0,0]^{T}$ is a column vector of order $15 \times 1$. Equation (18) denotes the set of fifteen algebraic equations which can be solved for $N$. Now comparison between exact solution and approximate solution of equation (15) is given in Table 2. 


\begin{tabular}{|c|c|c|c|}
\hline$t$ & $\begin{array}{c}\text { Hybrid Legendre polynomials } \\
\text { solution for } n=5, m=3\end{array}$ & Exact solution & Absolute error \\
\hline 0.0 & 1.99912 & 2.00000 & 0.00088 \\
0.1 & 1.63744 & 1.63746 & 0.00002 \\
0.2 & 1.34005 & 1.34064 & 0.00059 \\
0.3 & 1.09761 & 1.09762 & 0.00001 \\
0.4 & 0.89826 & 0.89866 & 0.00040 \\
0.5 & 0.73575 & 0.73576 & 0.00001 \\
0.6 & 0.60212 & 0.60239 & 0.00027 \\
0.7 & 0.49319 & 0.49319 & 0.00000 \\
0.8 & 0.40362 & 0.40379 & 0.00017 \\
0.9 & 0.33059 & 0.33060 & 0.00001 \\
\hline
\end{tabular}

Table 2. Comparison between approximate solution and exact solution for $k=2$ amd $m_{0}=2$

Also, equation (14) is solved for $k=1$ and $m_{0}=1$ and comparison between approximate solution and exact solution for $k=1$ and $m_{0}=1$ is shown in Table 3 .

\begin{tabular}{|c|c|c|c|}
\hline$t$ & $\begin{array}{c}\text { Hybrid Legendre polynomials } \\
\text { solution for } n=5, m=3\end{array}$ & Exact solution & Absolute error \\
\hline 0.0 & 0.99994 & 1.00000 & 0.00006 \\
0.1 & 0.90484 & 0.90484 & 0.00000 \\
0.2 & 0.81868 & 0.81873 & 0.00005 \\
0.3 & 0.74082 & 0.74082 & 0.00000 \\
0.4 & 0.67028 & 0.67032 & 0.00004 \\
0.5 & 0.60653 & 0.60653 & 0.00000 \\
0.6 & 0.54878 & 0.54881 & 0.00003 \\
0.7 & 0.49659 & 0.49659 & 0.00000 \\
0.8 & 0.44930 & 0.44933 & 0.00003 \\
0.9 & 0.40657 & 0.40657 & 0.00000 \\
\hline
\end{tabular}

Table 3. Comparison between approximate solution and exact solution for $k=1$ amd $m_{0}=1$

\subsection{Application of hybrid Legendre polynomials expansion in solving Hermite differen- tial equation of order zero}

Consider the Hermite differential equation of order zero (see [13])

$$
y^{\prime \prime}-2 t y^{\prime}=0
$$

with initial conditions

$$
y(0)=y^{\prime}(0)=1 \text {. }
$$

Now we have solved the equation (19) by hybrid Legendre polynomial operational matrix of integration for $n=5$ and $m=3$ given by (17), which is obtained by hybrid Legendre polynomial approximation method as below.

Let

$$
y^{\prime \prime}(t)=L^{T} h(t),
$$

where $L=\left[l_{10}, l_{11}, l_{12}, l_{20}, l_{21}, l_{22}, l_{30}, l_{31}, l_{32}, l_{40}, l_{41}, l_{42}, l_{50}, l_{51}, l_{52}\right]^{T}$ is $15 \times 1$ unknown column vector and $h(t)$ is also a column vector given by (16). Now expanding $f(t)=1$ and $g(t)=t$ by 
hybrid Legendre polynomials for $n=5$ and $m=3$, we obtain $f(t)=r^{T} h(t)$ and $g(t)=s^{T} h(t)$, where $r=[1,0,0,1,0,0,1,0,0,1,0,0,1,0,0]^{T}$ and

$$
s=\left[\frac{1}{10}, \frac{1}{10}, 0, \frac{3}{10}, \frac{1}{10}, 0, \frac{5}{10}, \frac{1}{10}, 0, \frac{7}{10}, \frac{1}{10}, 0, \frac{1}{10}, \frac{9}{10}, 0\right]^{T}
$$

are column vectors each of order $15 \times 1$. Now integrating equation (21) two times and using initial conditions given by (20), we find

$$
y^{\prime}(t)=L^{T} P h(t)+r^{T} h(t)
$$

and

$$
y(t)=L^{T} P^{2} h(t)+r^{T} P h(t)+r^{T} h(t) .
$$

Approximate $s^{T} h h^{T}$ by hybrid Legendre polynomials as

$$
s^{T} h h^{T}=h^{T} S,
$$

where $S$ is a square matrix of order 15 and it is given as

$$
S=\left[\begin{array}{ccccccccccccccc}
\frac{1}{10} & \frac{1}{10} & 0 & 0 & 0 & 0 & 0 & 0 & 0 & 0 & 0 & 0 & 0 & 0 & 0 \\
\frac{1}{30} & \frac{1}{10} & \frac{2}{30} & 0 & 0 & 0 & 0 & 0 & 0 & 0 & 0 & 0 & 0 & 0 & 0 \\
0 & \frac{2}{50} & \frac{1}{10} & 0 & 0 & 0 & 0 & 0 & 0 & 0 & 0 & 0 & 0 & 0 & 0 \\
0 & 0 & 0 & \frac{3}{10} & \frac{1}{10} & 0 & 0 & 0 & 0 & 0 & 0 & 0 & 0 & 0 & 0 \\
0 & 0 & 0 & \frac{1}{30} & \frac{3}{10} & \frac{2}{30} & 0 & 0 & 0 & 0 & 0 & 0 & 0 & 0 & 0 \\
0 & 0 & 0 & 0 & \frac{2}{50} & \frac{3}{10} & 0 & 0 & 0 & 0 & 0 & 0 & 0 & 0 & 0 \\
0 & 0 & 0 & 0 & 0 & 0 & \frac{5}{10} & \frac{1}{10} & 0 & 0 & 0 & 0 & 0 & 0 & 0 \\
0 & 0 & 0 & 0 & 0 & 0 & \frac{1}{30} & \frac{5}{10} & \frac{2}{30} & 0 & 0 & 0 & 0 & 0 & 0 \\
0 & 0 & 0 & 0 & 0 & 0 & 0 & \frac{2}{50} & \frac{5}{10} & 0 & 0 & 0 & 0 & 0 & 0 \\
0 & 0 & 0 & 0 & 0 & 0 & 0 & 0 & 0 & \frac{7}{10} & \frac{1}{10} & 0 & 0 & 0 & 0 \\
0 & 0 & 0 & 0 & 0 & 0 & 0 & 0 & 0 & \frac{1}{30} & \frac{7}{10} & \frac{2}{30} & 0 & 0 & 0 \\
0 & 0 & 0 & 0 & 0 & 0 & 0 & 0 & 0 & 0 & \frac{2}{50} & \frac{7}{10} & 0 & 0 & 0 \\
0 & 0 & 0 & 0 & 0 & 0 & 0 & 0 & 0 & 0 & 0 & 0 & \frac{9}{10} & \frac{1}{10} & 0 \\
0 & 0 & 0 & 0 & 0 & 0 & 0 & 0 & 0 & 0 & 0 & 0 & \frac{1}{30} & \frac{9}{10} & \frac{2}{30} \\
0 & 0 & 0 & 0 & 0 & 0 & 0 & 0 & 0 & 0 & 0 & 0 & 0 & \frac{2}{50} & \frac{9}{10}
\end{array}\right] .
$$

From the above we get

$$
\left(I-2 S P^{T}\right)=2 S r
$$

It is a system of algebraic equations which is solved for L. The exact solution of (19) is given by

$$
y(t)=1+\int_{0}^{t} e^{x^{2}} d x
$$

Comparison between approximate solution and exact solution is given in Table 4 . 


\begin{tabular}{|c|c|c|c|}
\hline$t$ & $\begin{array}{c}\text { Hybrid Legendre polynomials } \\
\text { solution for } n=5, m=3\end{array}$ & Exact solution & Absolute error \\
\hline 0.0 & 1.000 & 1.000 & 0.000 \\
0.1 & 1.101 & 1.100 & 0.001 \\
0.2 & 1.204 & 1.203 & 0.001 \\
0.3 & 1.311 & 1.309 & 0.002 \\
0.4 & 1.425 & 1.422 & 0.003 \\
0.5 & 1.548 & 1.545 & 0.003 \\
0.6 & 1.686 & 1.680 & 0.006 \\
0.7 & 1.840 & 1.833 & 0.007 \\
0.8 & 2.019 & 2.009 & 0.010 \\
0.9 & 2.229 & 2.215 & 0.014 \\
\hline
\end{tabular}

Table 4. Comparison between approximate solution and exact solution for $n=5$ and $m=3$

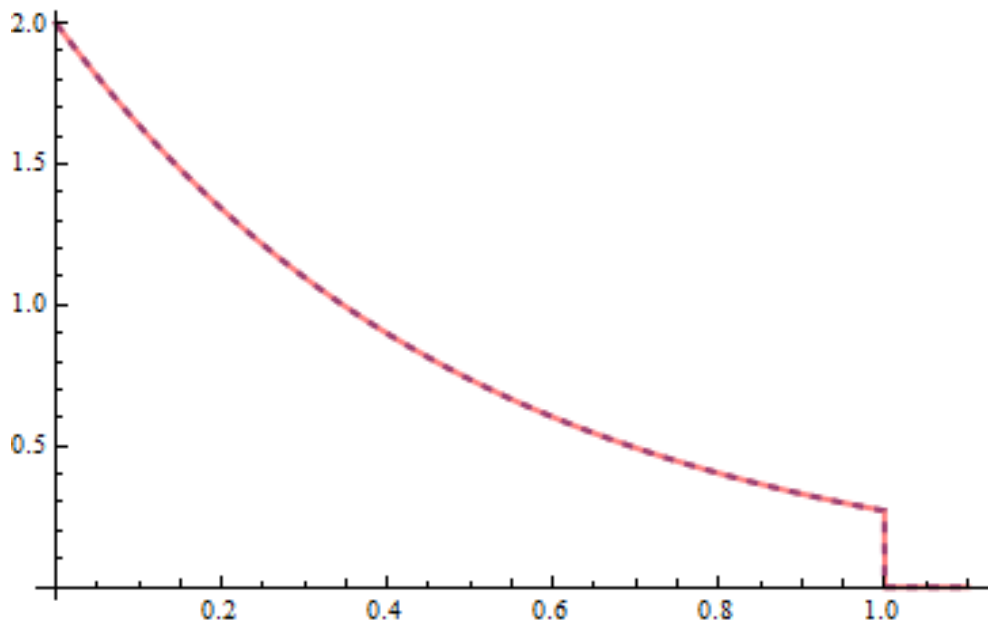

Figure 7. Graph of exact solution (dark line) and approximate solution (dashed line) of radioactive decay problem for $k=2$ and $m_{0}=2$

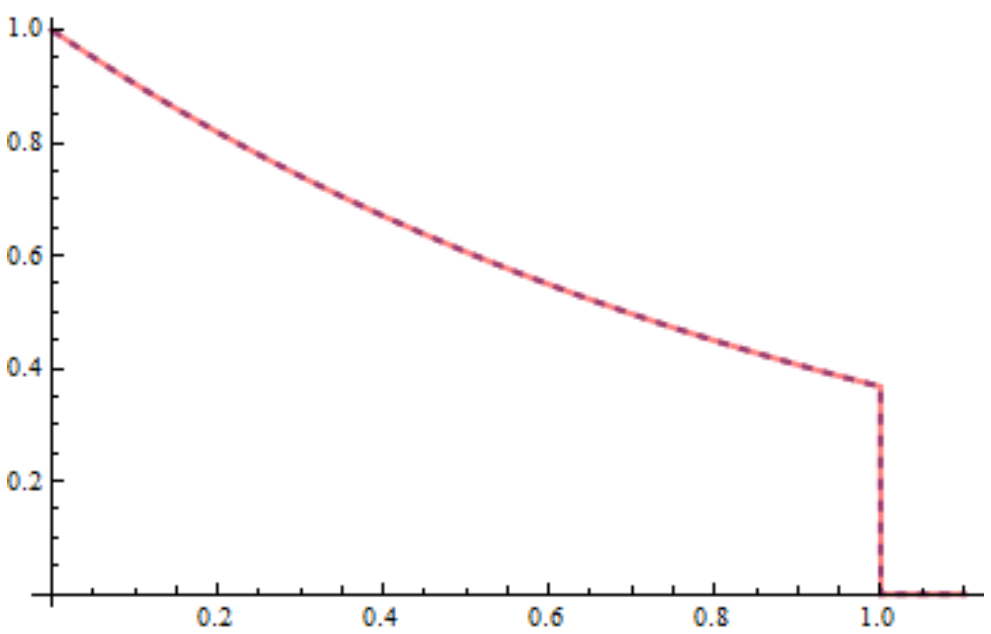

Figure 8. Graph of exact solution (dark line) and approximate solution (dashed line) of radioactive decay problem for $k=1$ and $m_{0}=1$ 


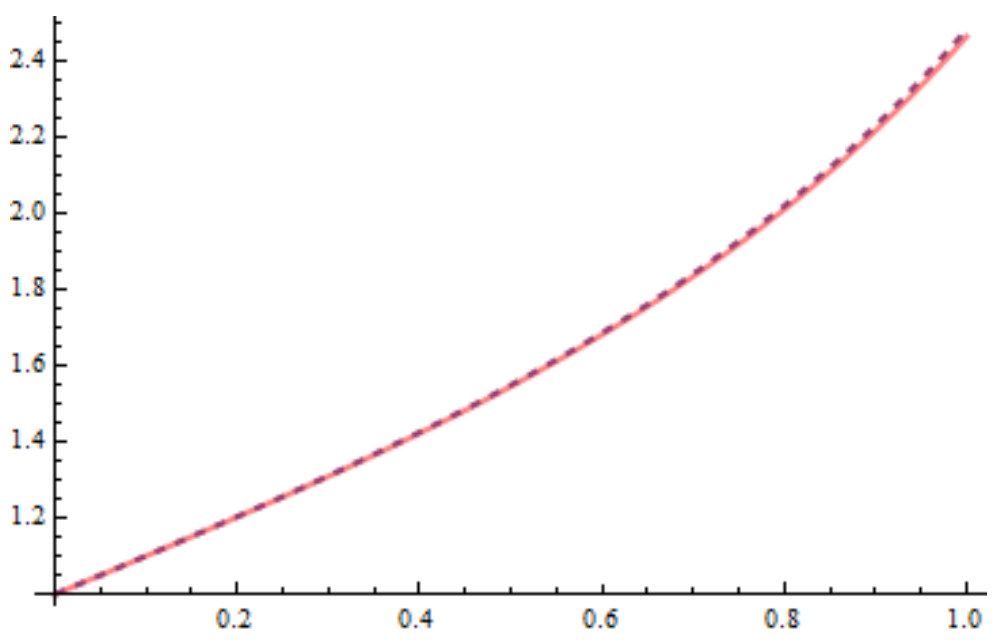

Figure 9. Graph of exact solution (dark line) and approximate solution (dashed line) of Hermite differential equation

\section{CONCLUSIONS}

1. Estimates of Theorems 1, 2 and 3 are given by

(i) $E_{m}^{(B P)}(f)=O\left[\frac{1}{m}\left(1+\frac{1}{m^{\alpha}}\right)\right]$;

(ii) $E_{m}^{(L P)}(f)=O\left[\left(1+\frac{1}{\sqrt{2 \alpha+1}}\right) \frac{1}{(2 m-3)^{\frac{3}{2}}}\right], \quad m \geq 2$;

(iii) $E_{n, m}^{(H F s)}(f)=O\left[\left(\frac{1}{n^{\alpha+2}}+\frac{1}{2 n^{2}}\right) \frac{1}{(2 m-3)^{\frac{3}{2}}}\right]$, where $m \geq 2,0<\alpha \leq 1$ and $n$ is a positive integer.

Since $E_{m}^{(B P)}(f) \rightarrow 0, E_{m}^{(L P)}(f) \rightarrow 0$ and $E_{n, m}^{(H F s)} f \rightarrow 0$ as $m, n \rightarrow \infty$, these approximations are best possible in wavelet analysis.

2. The solution of differential equations associated with the radioactive decay problem and the solution of the Hermite differential equation of order zero by hybrid Legendre polynomials is approximately same as the exact solution. This is the significant achievement of this paper.

\section{ACKNOWLEDGEMENTS}

Shyam Lal, one of the authors, is thankful to D.S.T(CIMS) for encouragement to this work.

Vivek Kumar Sharma, one of the authors, is grateful to U.G.C, New Delhi, India for providing financial assistance in the form of Junior Research Fellowship (JRF) vide letter no. 21.06.2015(i)EU - V(Dated - 05/01/2016).

Authors are grateful to the referee for his valuable comments and suggestions, which improve the quality and presentation of this paper. 


\section{REFERENCES}

[1] Abramowitz M., Stegun I.A. Handbook of mathematical functions with formulas, graphs, and mathematical tables. National Bureau of Standards, Applied Mathematics Series, 1965.

[2] Brychkov Y.A. Handbook of special functions: derivatives, integrals, series and other formulas. Chapman and Hall/CRC, 2008.

[3] Ebadian A., Khajehnasiri A.A. Block pulse functions and their applications to solving systems of higherorder nonlinear volterra integro-differential equations. Electron. J. Differential Equations 2014, 54, 1-9. http://ejde.math.txstate.edu

[4] Hsiao C.H. Hybrid function method for solving Fredholm and Volterra integral equations of the second kind. J. Comput. Appl. Math. 2009, 163 (1), 71-77. doi:10.1016/j.cam.2008.10.060

[5] Malik S.C., Arora S. Mathematical analysis. New Age International, 1992.

[6] Maleknejad K., Basirat B., Hashemizadeh E. Hybrid Legendre polynomials and Block-Pulse functions approach for nonlinear Volterra-Fredholm integro-differential equations. Comput. Math. Appl. 2011, 61 (2), $2821-2828$. doi:10.1016/j.cam wa.2011.03.055

[7] Maleknejad K., Mahdiani K. Iterated Block-Pulse Method for Solving Volterra Integral Equations. Applied Mathematics 2012, 2 (1), 17-20. doi:10.5923/j.am.20120201.03

[8] Marzban H.R., Razzaghi M. Numerical solution of the controlled duffing oscillator by hybrid functions. Appl. Math. Comput. 2003, 140 (2-3), 17-190. doi:10.1016/S0096-3003(02)00112-1

[9] Marzban H.R., Razzaghi M. Optimal control of linear delay systems via hybrid of block-pulse and Legendre polynomials. J. Franklin Inst. 2004, 341 (3), 279-293. doi:10.1016/j.jfranklin.2003.12.011

[10] Marzban H.R., Razzaghi M. Solution of multi-delay systems using hybrid of block-pulse functions and Taylor series. Journal of Sound and Vibration 2006, 292 (3-5), 954-963. doi:10.1016/j.jsv.2005.08.007

[11] Marzban H.R., Tabrizidooz H.R., Razzaghi M. Hybrid functions for nonlinear initial-value problems with applications to Lane-Emden type equations. Phys. Lett. A 2008, 372 (37), 5883-5886. doi:10.1016/j.physleta.2008.07.055

[12] Rai B., Choudhury D.P. Ordinary differential equations: an introduction. Alpha Science Int'1 Ltd, 2005.

[13] Saran N., Sharma S.D., Trivedi T.N. Special Functions. Pragati Prakashan, Meerut, 2014.

Received 29.05.2019

Revised 18.03.2020

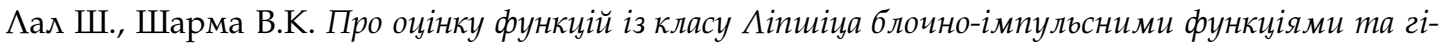
бридними поліномами Аежандра // Карпатські матем. публ. - 2020. - Т.12, №1. — С. 111-128.

У цій роботі, використовуючи блочно-імпульсні функції та гібридні поліноми Аежандра, знайдено оцінки функції $f$, яка має першу і другу похідні, що належать до класу Lip $[a, b]$, де $0<\alpha \leq 1$, i $a, b-$ скінченні дійсні числа. Отримані оцінки є новими, точними та найкрашими у вейвелет аналізі. Із метою пояснення обгрунтованості апроксимації функцій методом наближення гібридними поліномами Аежандра наведено приклад розв'язку задачі радіоактивного розпаду. Більше того, для пояснення важливості та застосування методики цього методу знайдено розв' язок диференціального рівняння Ерміта нульового порядку.

Ключові слова і фрази: блочно-імпульсна функція, поліном Аежандра, гібридний поліном Аежандра. 